\title{
Understanding the thermal expansion in layered perovskite chalcogenides
}

\section{J.M. Rondinelli}

Northwestern University, Evanston, IL USA

jrondinelli@northwestern.edu

Ferroelectric perovskite oxides have recently been used in solar applications because their polarity allows for the separation of photocarriers when under illumination to generate a photocurrent. Oxides, however, often have band gaps that are beyond the solaroptimal regime $(>3.3 \mathrm{eV})$; for this reason, perovskite-structured chalcogenides have been proposed as suitable candidate materials owing to their lower band gaps $(\approx 2 \mathrm{eV})$. An understanding of the thermal expansion behavior of photovoltaic materials is important so as to prevent large stresses and strains during fabrication and operation of the photovoltaic device. Here, we evaluate the structural, lattice dynamical, and thermodynamic properties of Ruddlesden-Popper chalcogenide $\mathrm{Ba}_{n+1} \mathrm{Zr}_{n} \mathrm{~S}_{3 n+1}(n=1,2,3, \infty)$ using the selfconsistent quasi-harmonic approximation within density functional theory [1]. These responses are compared to the thermal expansion of Ruddlesden-Popper oxides and recent experimental data, which allows us to suggest guidelines for engineering thermal expansion in the Ruddlesden-Popper structure type with diverse chemistries.

[1] Koocher, N. Z., Huang, L.-F. \& Rondinelli, J. M. (2021). Phys. Rev. Materials 5, 053601.

Keywords: Thermal expansion; high pressure; Ruddlesden-Popper; DFT

This work was supported by the National Science Foundation's MRSEC program (DMR-1720139) at the Materials Research Center of Northwestern University. 